\title{
BUILDING REAL LABORATORIES ON THE INTERNET
}

\author{
Kyle Forinash, Raymond Wisman
}

\begin{abstract}
A majority of the pedagogical uses of computers fall somewhere within the five headings of Drill, Number Crunching, Laboratory applications, Simulations, and Self-paced courses [2]. In this paper we discuss a sixth use for computerized instruction, dispersed laboratories over the Internet, one which was probably unanticipated years ago when the Internet and laboratory technologies were less accessible and less friendly than now. The pervasive interconnectivity of the Internet provides the means for entirely new kinds of laboratory experiences for the science student. For example, students can remotely access laboratory equipment that previously would have been too expensive or too dangerous for a typical student laboratory. Large-scale collaborative experiments between students separated geographically are also possible. Our efforts have been to adapt familiar laboratory and Internet technologies to support relatively easy faculty authorship of lessons and foster scientific exploration by the student. One outcome has been a straightforward and general solution using the Excel spreadsheet application for faculty lesson authorship and student data collection and analysis from shared instruments over the Internet. This paper presents an overview of the technology for science laboratories over the Internet, example laboratory experiments, and potential applications.
\end{abstract}

\section{KEYWORDS}

Internet laboratories, spreadsheets, computer-based instruction

\section{INTRODUCTION}

In 1984, A. B. Arons in a paper on computer-based instructional dialogs in science courses [2], listed five uses of computers for instructional purposes in the sciences; Drill, Number Crunching, Laboratory applications, Simulations, and Self-paced courses. Although 18 years has passed (a long time in the computer world), his comments and criticisms of these pedagogical uses of computers hold up quite well. Probably the most significant change in computing since that paper was written has been the Internet and the new kinds of interactions and collaborations between people that are now practical. One well-known example of the Internet's potential is Napster [10], one of many programs that create a vast group collection from individual contributions scattered across the Internet. An individual using Napster to find music also automatically shared music files on their computer with others, creating a collaborative snowball-effect that increased the collection size to the benefit of all users. Many other models are possible that exploit the potential for collaboration and distribution of resources over the Internet. In this paper we present several simple and inexpensive designs for computer-based experiments in undergraduate physics and computer science laboratories and examine the potential for large-scale collaboration and sharing of data collection resources.

Computer-aided experimentation has a long and venerable history in many scientific areas, particularly physics and astronomy. Most high school and college physics labs already control experiments, collect and analyze data using laboratory computers (see for example [12]). Remote data collection with the aid of computers has also been used in physics and astronomy for some time; the Martian rover and the Hubble space telescope are two notable examples of data collection at a distance. Until recently, remote 
data collection generally required significant investments of resources and infrastructure. With the Internet, not only is remote data collection easy and cheap enough for student laboratories, it also offers the possibility of new kinds of laboratory experiences.

Our main goal has been to develop inexpensive and easy to use computer-based laboratory experiments in introductory physics and computer science networking courses. A secondary goal has been to deliver the experiments directly to the student on demand and make it possible for students to perform experiments remotely, such as from their home. Our approach naturally supports sharing of laboratory equipment, remote data collection, and collaboration between users over the Internet. One important point we would like to emphasize is that the computer-based laboratory experiments we are presenting are real experiments rather than simulations. We also do not believe that computer-based experiments should replace traditional laboratory experiments or that our approach is appropriate for all computerbased laboratories. However, science education should include experiments that may require a computer when experimental events occur too rapidly, are too dangerous, or too distant for a student to directly perform. We feel the student laboratory experience can also be enriched using the Internet to provide collaboration and collecting data from multiple sources.

In the discussion below we give some arguments for genuine experiments and specific examples of combining the Internet with other common technologies to perform experiments. In our conclusion we will speculate on the future of the Internet and computer-based experiments in science education.

\section{WHY REAL EXPERIMENTS ARE IMPORTANT}

Experimentation is clearly central to scientific investigation but what compelling reasons are there for student experiments, particularly the textbook exercise in which the results are already well known? There are two main elements to an experiment: design of the experiment to test a hypothesis and analysis of data from the experiment. No experiment can be performed with zero error, so one must determine with what degree of certainty the data supports a particular hypothesis. Coming to terms with the inaccuracy and imprecision of results requires knowledge of the interplay between experimental design and data analysis. Some laboratory skills, such as statistical analysis of data, can be learned in the abstract, outside of the laboratory. Experimental design, however, can only be learned from using real equipment in real experiments, often through a certain amount of trial and error. It should be no great surprise that student practice of experimentation is needed to understand science, that educational abstractions alone are not enough. Education, sports, and life present many parallels, for who has mastered even the rudiments of arithmetic, tennis, or driving without many mistakes and considerable practice? In addition to other laboratory goals (see references [1], [9] and [11] for example) it is crucial that this skill, the art of experimental design, be communicated to the student through the genuine laboratory experience.

Without an understanding of experimental design, one can easily be misled by data, even data that is technically error free. All experimenters have experience with sampling rate errors inherent in data collection. For example, a sinusoidal voltage signal sampled by chance too slowly at each wave maximum would lead the naïve experimenter to conclude the signal was DC. Recognition of experimental design and procedural errors are a crucial feature of any student laboratory experience intending to educate students as critical investigators. If the student is not faced with a situation where they have to "trouble shoot" and "experiment" with the design, procedures and data collection parameter space of the experiment, the student has not been properly challenged and the laboratory experience is deficient. For example, if the experiment requires sampling a simple sine wave the student should leave the laboratory being able to answer such questions as: 1) How often should the signal be sampled in order to make a proper determination of the signal frequency? 2) Is a sample rate of twice the signal frequency fast enough? 3) Does the hypothesis under investigation require being able to determine even smaller oscillations calling for a much faster sample rate? 4) Is the equipment capable of sampling at a faster rate? In the sinusoidal voltage signal example, the error was not in the equipment 
but rather in the experimental design and procedure. A single choice in experimental parameters, the sample rate in this case, can produce completely spurious results.

\section{GOALS FOR COMPUTER-BASED LABORATORY DELIVERY}

The authors have used computers for in-residence student laboratories for many of the years since Aaron's paper and for several years have incorporated the Internet as a delivery tool for selected laboratory exercises performed remotely. This experience has indicated three key requirements that must be satisfied for a remote laboratory to compare favorably with an in-residence laboratory: 1) students must have enough control of remote lab equipment to start and stop an experiment and make appropriate adjustments to experimental parameters, 2) the remote experiment should be no more difficult to conduct than with the equipment physically present, and 3) students need appropriate feedback regarding the progress of an experiment. By satisfying these requirements the two key elements of experimental design and error analysis, important in a traditional laboratory, remain viable elements of laboratories delivered over the Internet.

It can also be argued that providing Internet access to remote laboratory equipment can provide a superior educational experience compared to a purely in-residence laboratory by transcending boundaries of time and place. In the following, we offer observations of some advantages to distance laboratories.

Student access - The typical science undergraduate laboratory meets three hours once a week. This rigid format requires synchronized attendance by instructor and all students, yet yields a very short period of laboratory access. Laboratory exercises made available over the Internet have the potential to provide constant access whenever needed by students.

Safety - Safety issues limit the kinds of laboratory experiences available to undergraduate science students. Examining various radioactive sources is an experiment that might be considered moderately hazardous and is often avoided in an introductory setting or limited to weak radiation sources for safety. High powered lasers and X-ray sources are tools used for research but are considered too dangerous for introductory students. Such safety concerns can be diminished when the experiment is distant to the experimenter.

Variety of experiments - The variety of laboratory exercises a student may encounter is limited by the rigid time and place format of the traditional laboratory setting. Experiments that require monitoring longer than the laboratory class time are seldom done. Experiments that monitor geographically distant phenomena, such as atmospheric and seismographic data, or that require multiple collection sites are generally avoided because of logistical difficulties. Internet accessed labs can expand the possibilities of the traditional lab by freeing the experiment from time and location constraints.

Cost - Certain laboratory equipment is so expensive that it is difficult to purchase and maintain the multiple stations needed in a traditional science laboratory. Providing sufficient equipment access in a traditional lab requires a trade off between buying more equipment, which is expensive, and asynchronous student use, which requires additional laboratory staffing with increased expense. Such problems can be mitigated by extended student access to laboratory equipment, requiring fewer lab stations and realizing an equipment savings. Several examples exist of student controlled laboratory experiments available on continual, asynchronous basis (for example the gamma-ray spectrometer online at the University of Tennessee [6]).

Ideally, the computer is a natural piece of the experimental equipment; at a minimum the computer should not make the purpose of the experiment less obvious. We have experimented with several user interfaces as seen from our examples below. The most intuitive and technologically simplest approach for computed-based laboratories explored to date has been to combine the standard spreadsheet application with network access to instruments so that the experiment may be near or remote to the 
experimenter. Spreadsheets have long been used for the data analysis of experiments [12]; flexible but relatively easy to learn, they intrude lightly when used to perform the experiment. Other, more potent software can be used but generally at an increase to the implementation cost for faculty and learning time for students. The key is to minimize faculty time and skills in developing a worthwhile laboratory experiment and student computer skills in performing the experiment. The spreadsheet, when adapted to perform the remote or local experiment in addition to normal data analysis, provides a simple, easily understood tool for faculty and students.

For the instructor, advantages of using a spreadsheet tool are the low skill level and effort needed to create a lab since the spreadsheet is familiar and relatively easy to use. To create a lab, the instructor might arrange a few of the preliminary lab steps, such as how to get data from an instrument into the spreadsheet, leaving the remaining steps of performing the experiment and data analysis to the student. A student can then open the same spreadsheet from a Web page at home or in the traditional lab setting and essentially pick up where the instructor stopped. Instructions, graphics, video or audio can also be provided with the spreadsheet on the Web page. Spreadsheets have long been used in science laboratories to analyze data but require only some (surprisingly!) minor programming to access instruments. Technical details on the necessary programming is given in references [4] and [15].

\section{EXAMPLES OF THE INTERNET AS A LABORATORY TOOL}

We present four laboratory examples in which our students have used computer-based labs and describe other cases that survey the potential of the Internet as a laboratory tool. The instrumentation required is relatively inexpensive, well within the reach of most high school and introductory college laboratories (less than $\$ 300$ each [14]). Many school laboratories use similar equipment for in-residence labs. Note that all examples can be implemented with a standard spreadsheet, although some were initially done as a computer program before recognizing the spreadsheet potential for computer-based laboratories.

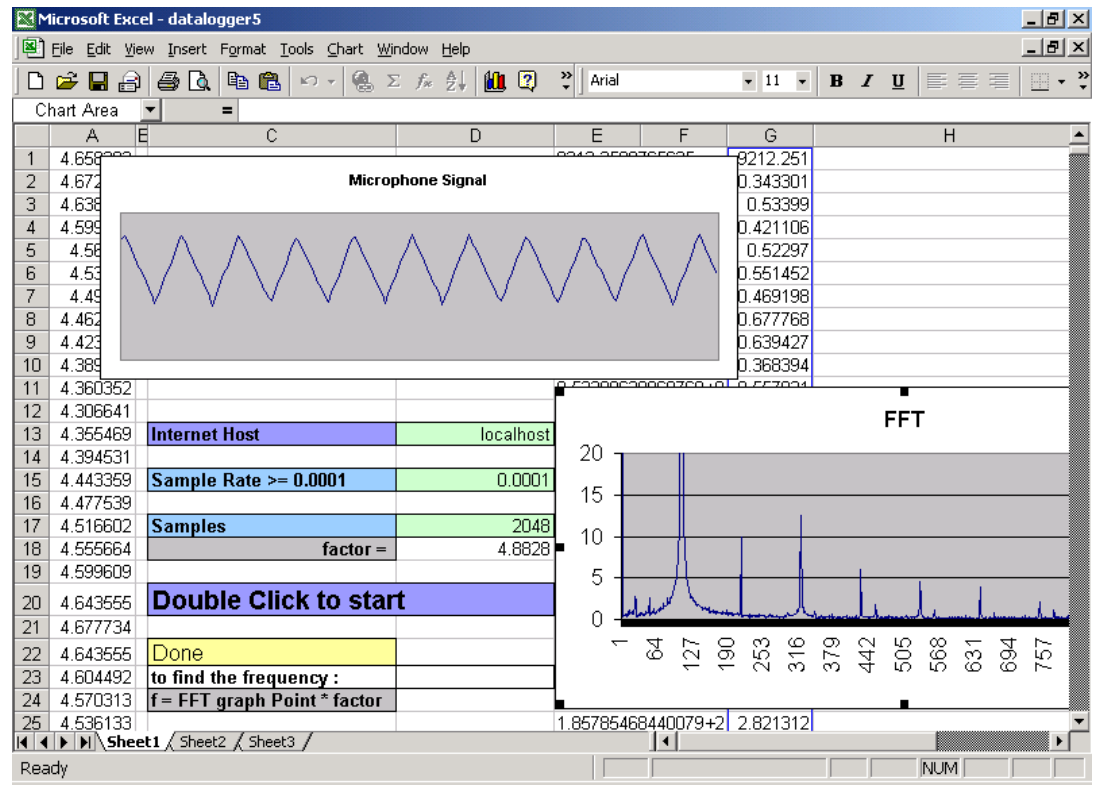

Figure 1. A spreadsheet used in a physics lab to analyze the frequencies present in a triangle wave. The student types the Internet Host address of the computer with attached instruments (localhost, meaning the local computer in this example), sets the sample rate and the number of samples and double clicks to collect data (shown in the upper left graph). The student then uses spreadsheet analysis tools to analyze the data the frequencies present (lower graph labeled FFT). To collect data from a remote host the student need only replace 'localhost' with the remote host Internet address.

The first example follows the typical scheduled laboratory model covering a physics experiment in which students study the frequency component of sound while playing musical instruments, singing, or 
otherwise making noise. The experiment connects the students' intuitions of sounds heard in the lab with precise measurements of frequency and waveforms. In the experiment, sound waves are received by a microphone, digitized, and made available over the Internet. Students begin the experiment with an instructor prepared spreadsheet that collects and graphs the raw digital sound data as they make noise (see Fig. 1). The spreadsheet tools include Fourier analysis and graphics needed to analyze the frequency spectrum of the sound data. Students directly control experimental parameters, such as sample rate and sound source, by changing appropriate cells of the spreadsheet. The full lab instructions and spreadsheet are part of a Web page and can be used from any computer with the spreadsheet application and an Internet connection [5].

The sound lab is conducted in a traditional setting, during a scheduled time with the instructor present, with the computer, apparatus, musical sound sources, and students all at the same location. However, in addition to sounds made by students in the lab room, an unknown sound source located in another room is included for students to analyze. By changing one cell in the spreadsheet to the distant device Internet address, students collect data from the 'unknown' distant sound source. Determining the frequency of the unknown source requires that students experiment with the measurement sample rate and the number of data points collected. As part of the laboratory report, students explain the accuracy and error inherent in the measurements, requiring an understanding of measurement instrument limitations, experimental design, and the spreadsheet tools used to analyze the data. Advantages realized using the spreadsheet approach are the very low development effort for the instructor and the opportunity for students to follow an investigative approach not anticipated by the instructor.

A second example presents a model where students use laboratory equipment at school, from home, or elsewhere over the Internet. In this example, students in a computer science course on networking observe signals of a digital serial data transmission. The students are asked to transmit a message and then determine characteristics such as transmission rate, amplitude, original data transmitted, and framing and data checking information from the observed signal.

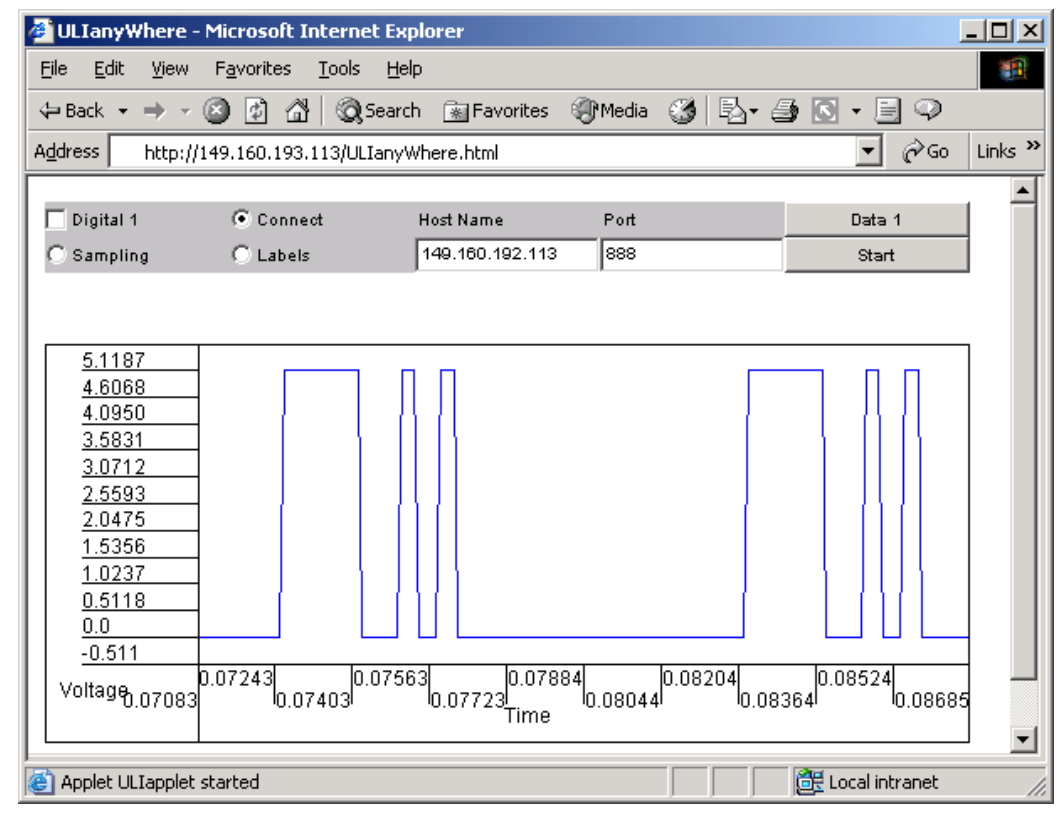

Figure 2. A Java-applet oscilloscope displaying a digitized signal of a serial data transmission

Students transmit text to a computer with an analog-to-digital converter (i.e. Host Name of 149.160.192.113) and analyze the corresponding signal captured on the oscilloscope. The display presents two ASCII encoded characters 'X' of binary 01011000 with data, start, stop, and parity bits transmitted at 2400 baud. A binary 1 corresponds to voltage 0 and a binary 0 to voltage +5 . 
This example began life as a traditional laboratory with equipment consisting of one computer to output data forming the signal, an analog-to-digital converter instrument to measure the signal, and another computer for collecting and graphically displaying the signal in the manner of an oscilloscope. Although there was no scheduled laboratory time, students often queued for access to the single equipment setup that was literally wired together into a single unit. In true educational tradition, we developed a replacement laboratory using remotely accessed equipment mainly for presenting a networked application example and not directly to benefit waiting students. The new laboratory arrangement bundled a Java-applet oscilloscope (see Fig. 2) and instructions onto a Web page with analog-to-digital converter access through a laboratory computer connected to the Internet. Although two computers were still required, students could transmit messages to the single analog-to-digital converter and analyze results from any computer. As an unintended benefit, because any one student used the analog-to-digital converter only a few seconds throughout the assignment and could be asynchronously shared among all students, the single analog-to-digital converter was sufficient. Also unexpected but in hindsight not surprising, although we expected and planned for students to use laboratory computers, a number of students completed the assignment from home.

The strongest argument against developing laboratories using Java-applets or some other programming language is the time and skill required to craft the tools used by the student. The Java-applet required between one and two orders of magnitude more time to develop than the more sophisticated and flexible spreadsheet-based laboratory of our first example.

One potential benefit of laboratories available on-line is new science education models which ignores time and place. The first example above presented the traditional model of each student with one local equipment setup used during a regular laboratory time. The second example presented a different model where multiple students asynchronously used one distant equipment setup at their convenience. Our third example presents a model where multiple students used one remote data collection device simultaneously. The equipment setup consisted of three antennas (used in a student research project based on a Scientific American column [3]) designed to receive extremely low frequency electromagnetic signals. These were buried at the edge of our campus making direct student and instructor access physically difficult. In this case, students could not change instrument parameters but could only collect and analyze the signal data, so that an observation by one student using the same instrument did not interact with an observation by another. This model would be generally useful where many students monitored equipment that is otherwise inaccessible, such as remote weather stations.

Distributed data collection via the Internet also offers unique opportunities for educational models that take advantage of the non-localized character of the Internet, as explored in the fourth and final example. As part of a project, one student used multiple instrument setups simultaneously over an uninterrupted one-week period. Several volunteers at sites in Nebraska, New York, Indiana and Pennsylvania attached a light sensing instrument to their Internet connected computer and ran a small communications program to give access to the instrument. The communications program did not impair the normal local activities of the computer at the remote locations. Over the week, the student's computer program controlled instrument parameters and recorded the light measured at each distant site every second from our campus. With inexpensive instruments and technically simple means, other data could easily be collected from multiple distant sites. One unanticipated discovery was the logistics of locating and coordinating distant volunteer sites was far more challenging than the technical elements of the project. The current limits of using the Internet to perform large-scale, distant laboratory exercises seems more dependent on limitations of human resourcefulness than with technical challenges.

\section{CONCLUSION}

Shared data collection over the Internet offers the possibility for very different kinds of learning opportunities, for example large-scale collaboration. There are several projects involving schools around the world that share local data collected by hand, for example The Globe Program for primary 
and secondary school students [7]. Other existing projects collect data directly through computers connected to sophisticated equipment but require significant software and hardware development. Two such projects are the 'Innovative Educational Concept for Autonomous and Teleoperated Systems' project linking several European and North American sites to share robotic equipment for training engineering students [8] and the sharing of telescope access through the 'Share Your Sky' program [13].

We believe that networked laboratories offer unique educational opportunities but must be simple and inexpensive for instructors to develop while clear and flexible for students to use. We offer our experience using spreadsheets as a possible approach. With minimal development, experiments based on sharing local weather data of barometric pressure, temperature and wind speed are quite possible. Other collaborations, such as seismographic data, ambient particulate contamination, air quality in different parts of a building or in an entire organization, solar intensity levels, changes in local magnetic flux, local radiation levels, $\mathrm{CO}_{2}$ levels, changes in local gravitational acceleration and oxygen levels over wide geographic regions become very feasible. One can only imagine the results if students had been able to monitor local radiation levels in various parts of Europe after the Chernobyl incident or the nuclear tests in Pakistan and India. Such projects appear to require large amounts of equipment and organization. However, with the Internet as a collaborative tool and cooperation at the distant sites, as described here, it is quite practical to perform some of these collaborations cheaply and effectively.

While we do not believe distance laboratories can or should replace other approaches entirely, laboratory equipment made available through the Internet can offer considerable educational advantages, even when compared to traditional laboratories. Greater student access can be provided to equipment that would not otherwise be available because of time restrictions of the student or the instructor. Physical location and expense of the equipment can be made less important. Web-based delivery also offers a means to conveniently package a complete laboratory that includes written guides, data collection, and data analysis tools, whatever the location of student or experiment. And finally, by keeping control of a real experiment in the hands of the student, distance laboratories can achieve the same educational goals important in the traditional laboratory.

\section{REFERENCES}

AAPT, "Goals of the Introductory Physics Laboratory." The Physics Teacher 35, 546-548 (1997).

A. B. Arons; "Computer-Based Instructional Dialogs in Science Courses" Science Vol. 224, No. 4653 (1984) p1051.

Shawn Carlson; The Amateur Scientist, Scientific American, May (1996) p98.

K. Forinash, R. Wisman; "Simple Internet data collection for physics laboratories" American Journal of Physics Vol. 70, No. 4 (2002) p458.

K. Forinash, R. Wisman; The spreadsheet can be downloaded directly from http://Physics.ius.edu/ kyle/P222/FFTlab.html (change 'localhost' to physics.ius.edu in the spreadsheet and double click to receive data from a signal generator).

Gamma Ray Spectroscopy http://electron5.phys.utk.edu/gamma/Default.htm

The Globe Program: http://www.globe.gov/globe_flash.html

Innovative Educational Concept for Autonomous and Teleoperated Systems: http://www.ars.fhweingarten.de/iecat/iecat.html

Lazarowitz and Tamir, "Research on using laboratory instruction in science." in Handbook of Research on Science Teaching and Learning, edited by D. Gabel (MacMIllan Publishing Company, New York NY, 1993). 
Napster. http://www.howstuffworks.com/napster1.htm

Rief and St. John, "Teaching physicists' thinking skills in the laboratory." American Journal of Physics 47, 950-957 (1979).

E. F. Redish and J. S. Risley, Editors; The Conference on Computers in Physics Instruction Proceedings (Addison-Wesley Publishing Company, Redwood City, CA, 1990).

Share Your Sky: http://shareyoursky.com/

Vernier Software, 13979 SW Millikan Way, Beaverton, OR 97005-2886.

R. Wisman, K. Forinash; Sample software including an Excel spreadsheet to control a LabPro can be downloaded from http://Physics.ius.edu/ kyle/K/DataCollect/LabPro.html

Kyle Forinash

School of Natural Sciences

Indiana University Southeast

New Albany, IN 47150, USA

Email : kforinas@ius.edu

Raymond Wisman

School of Natural Sciences

Indiana University Southeast

New Albany, IN 47150, USA

Email: rwisman@ius.edu 